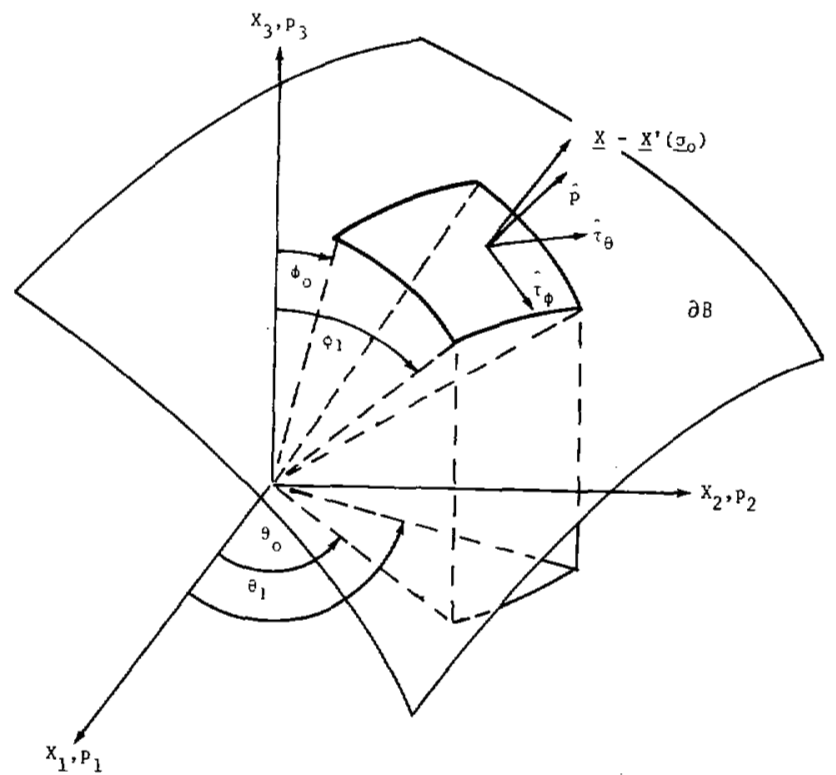

Fig. 3. Three-dimensional view of target surface and aperture field.

\section{CONCLUSIONS}

The physical optics inverse scattering problem has been shown to be tractable, even with the absence of low-frequency data, or data measured from all aspect directions. Indeed, the method presented here yields better resolution when data does lie in the higher-frequency regimes. This analysis shows that at least the reconstruction of specular reflection points is possible from high-frequency band-limited data. Lewis [2] and Perry [3] have examined an integral equation approach to the problem of fully reconstructing the characteristic function $\gamma(\mathrm{X})$. It is believed that the results produced by this method would provide a satisfactory starting point in an iteration scheme for solving that integral equation.

\section{REFERENCES}

[1] N. N. Bojarski, "A survey of Electromagnetic Inverse Scattering," Syracuse Univ. Res. Corp., Special Projects Lab. Rep., Oct. 1966, DDC\#AD-813-581.

[2] R. M. Lewis, "Physical optics inverse scattering," IEEE Trans. Antennas Propagat., vol. AP-17, pp. 308-314, May 1969.

[3] W. L. Perry, "On the Bojarski-Lewis inverse scattering method," IEEE Trans. Antennas Propagat. (Commun.), vol. AP-22, pp. 826-829, Nov. 1974.

[4] W. Tabbara, "On an inverse scattering method," IEE Trans. Antennas Propagat. (Commun.), vol. AP-21, pp. 245-247, Mar. 1973.

[5] -, "On the feasibility of an inverse scattering method," IEEE Trans. Antennas Propagat. (Commun), vol. AP-23, pp. 446448, May 1975.

[6] S. Rosenbaum-Raz, "On scatterer reconstruction from far-field data," IEEE Trans. Antennas Propagat, (Commun.), vol. APpp. 66-70, Jan. 1976.

[7] N. Bleistein and R. A. Handelsman, Asymptotic Expansions of Integrals. New York: Holt, Rinehart and Winston, 1975.

\title{
Remote Sensing of Sea State by Analysis of Backscattered Microwave Phase Fluctuations
}

\author{
S. F. CLIFFORD AND D. E. BARRICK, MEMBER, IEEE
}

\begin{abstract}
The relative phase of a normally incident microwave signal scattered off the sea surface is a random function of space and time. The statistics of these random phase fluctuations contain information about the wave-height statistics. This study demonstrates that the wave-height spectra can be deduced directly in terms of the spectra of the phase fluctuations without recourse to inversion techniques even when surface roughness exceeds many wavelengths of the incident signal. In the particular case of a nadir-directed satellite-mounted microwave source operating in the backscatter mode, the wave-height and phase spatial spectra are proportional with a constant proportionality of $\left(2 k_{0}\right)^{2}$, where $k_{0}$ is the wavenumber of the scattered signal.
\end{abstract}

Manuscript received March 19, 1977; revised February 15, 1978. This work was supported in part by a grant from NASA/Wallops Island, order number P-67,583 (G).

The authors are with the Wave Propagation Laboratory, Environmental Research Laboratories, National Oceanic and Atmospheric Administration, Boulder, CO 80302.

\section{INTRODUCTION}

M ANY techniques have been suggested for deriving knowledge of the sea state from both passive and active electromagnetic observations. These include various high-frequency (HF) scatter techniques [1], [2], the laser profilometer [3], the short pulse microwave altimeter [4], and the microwave scatterometer [5]. We propose a new technique that obtains direct information about sea state parameters from analysis of the phase fluctuation spectrum of a microwave signal reflected off the sea surface. The technique requires an airplane-borne or satellite-mounted microwave source that illuminates the sea surface at normal incidence and detects the backscattered return. By applying the theoretical model of scattering that found such useful application to the problem of optical and microwave propagation in random media, we are able to derive 
the wave-height spectrum directly in terms of the spectrum of backscattered phase fluctuations.

\section{ANALYSIS}

From existing theoretical formulations based on physical optics [6], [7] we can derive the following equation for the magnetic field backscattered from a perfectly conducting ocean surface for a normally incident spherical wave

$$
H\left(\rho^{\prime}\right)=\frac{-i k_{0} \mathrm{C}_{0}}{2 \pi} \int_{A} d^{2} \rho^{\prime} \frac{e^{i 2 k_{0} r} \sec \theta}{r^{2}},
$$

where $k_{0}$ is the wavenumber of the incident radiation, $\mathbf{C}_{0}$ is the incident polarization vector of the magnetic field, and $r$ is the distance from the scattering point $P$ to the point of observation $P^{\prime}$ (see Fig. 1). The origin of coordinates is at $\left(\rho^{\prime}, 0\right)=$ $\left(x^{\prime}, y^{\prime}, 0\right) ; r=\sqrt{\rho_{1}^{2}+\left[z^{\prime}-\xi\left(\rho_{1}+\rho^{\prime}\right)\right]^{2}} ; \xi$ is the wave height at $P, \theta$ is the angle from the nadir to the scattering point $P ; A$ is the illuminated area, and $z^{\prime}$ is the height of the transmitterreceiver above the mean sea surface. To a close approximation $z^{\prime}$, the height of the transmitter, can replace $z^{\prime}-\xi$ in $\sec \theta / r^{2}$; however, in the phase we must retain $\xi$ because we assume that the wave-height variations are much greater than a wavelength of the microwave signal. These approximations give

$$
\mathbf{H}\left(\rho^{\prime}\right)=\frac{-i k_{0} \mathrm{C}_{0}}{2 \pi z^{\prime}} \int_{A} d^{2} \rho_{1} \frac{e^{i 2 k_{0} \sqrt{r_{0}^{2}-2 z^{\prime} \xi+\xi^{2}}}}{r_{0}},
$$

where $r_{0}=\sqrt{\rho_{1}^{2}+z^{\prime 2}}$.

We now introduce the angle $\theta_{1}$, which is the angle from the nadir to the mean sea surface plane beneath the point $P$, and expand the argument of the exponential in terms of $\theta_{1}$,

$$
\begin{aligned}
2 k_{0} \sqrt{r_{0}^{2}-2 z^{\prime} \xi+\xi^{2}}= & 2 k_{0}\left[\left(r_{0}-\xi\right)^{2}\right. \\
& \left.+2\left(1-\cos \theta_{1}\right) \xi r_{0}\right]^{1 / 2} \\
\approx & 2 k_{0}\left(r_{0}-\xi\right)\left[1+\frac{\theta_{1}^{2} \xi r_{0}}{2\left(r_{0}-\xi\right)^{2}}\right. \\
& -\cdots],
\end{aligned}
$$

where we have approximated $\left(1-\cos \theta_{1}\right) \sim 1-\theta_{1}{ }^{2} / 2$ which is valid if $\theta_{1} \ll 1$. Since $\theta_{1} \leqslant \theta_{0}$ where $\theta_{0}$ is the half beamwidth, assumed to be less than a few degrees, this approximation is reasonable. The second term in (3) may be ignored if

$$
\frac{k_{0} \theta_{1}^{2} \xi_{0}}{\left(r_{0}-\xi\right)} \sim k_{0} \theta_{0}^{2} \xi_{\mathrm{rms}} \ll 1 .
$$

For a typical microwave signal $(\lambda=3 \mathrm{~cm}) k_{0} \sim 200 \mathrm{~m}^{-1}$ and for a rough sea surface $\xi_{\mathrm{rms}} \sim 1 \mathrm{~m}$, the approximation is valid if $\theta_{0}<4^{\circ}$. With this approximation applied to (3) we obtain the final working equation

$$
\mathbf{H}\left(\rho^{\prime}\right)=\frac{-i k_{0} \mathbf{C}_{0}}{2 \pi z^{\prime}} \int_{A} d^{2} \rho_{1} \frac{e^{i 2 k_{0}\left[r_{0}-\xi\left(\rho_{1}+\rho^{\prime}\right)\right]}}{r_{0}} .
$$

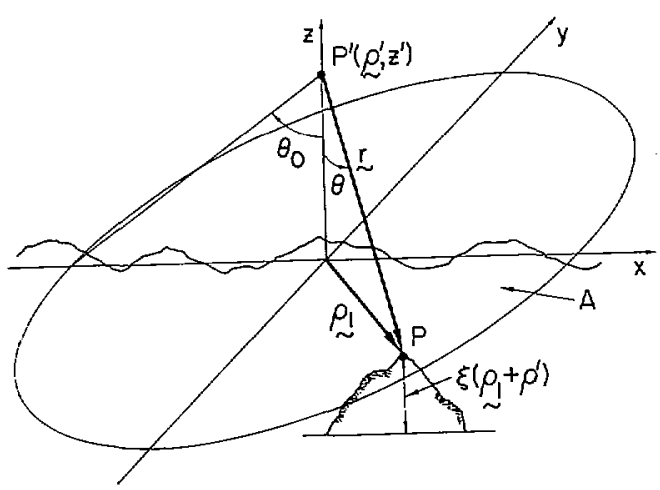

Fig. 1. Geometry of seascatter. Satellite source with beamwidth $\theta_{0}$ at $P^{\prime}$ illuminates surface area $A$. Signal is returned from waveheight perturbation $\xi$ at point $P$.

Because polarization fluctuations are ignored in the formulation, ( $C_{0}$ is a constant), we can drop the vector character of the scattered field and deal with the scalar $H$ instead of $\mathbf{H}$. The first steps are to normalize $H$ to $H_{0}$, the field that would be received if the sea were perfectly calm, and define $\gamma\left(\rho^{\prime}\right)=$ $H\left(\rho^{\prime}\right) / H_{0}$. The dependence of $\gamma$ on the amplitude and phase fluctuations of the backscattered field can be explicitly shown by writing $H$ and $H_{0}$ in phasor notation, $H=A e^{i \phi}$ and $H_{0}=$ $A_{0} e^{i \phi_{0}}$. The total field $H$ is a random variable whose amplitude and phase have both mean $A_{0}$ and $\phi_{0}$ and fluctuating parts $A_{1}$ and $\phi_{1}$; consequently,

$$
\gamma=\left(A / A_{0}\right) e^{i\left(\phi-\phi_{0}\right)}=\left(1+A_{1} / A_{0}\right) e^{i \phi_{1}}
$$

If we ignore the normalized amplitude fluctuations with respect to the enormously larger phase fluctuations, which is a reasonable approximation in the case of scattering from the sea surface (see Appendix), the fluctuations in $\gamma$ can be entirely attributed to phase fluctuations, i.e.,

$$
\gamma\left(\rho^{\prime}\right) \cong e^{i \phi_{1}\left(\rho^{\prime}\right)}
$$

The problem remaining is to relate the statistics of $\gamma$ to the wave-height statistics by analysis of (5).

Consider the equation for $\gamma$,

$$
\gamma\left(\rho^{\prime}\right)=\frac{-i k_{0} C_{0}}{2 \pi z^{\prime} H_{0}} \int_{A} d^{2} \rho_{1} \frac{e^{i 2 k_{0}\left[r_{0}-\xi\left(\rho_{1}+\rho^{\prime}\right)\right]}}{r_{0}} .
$$

Both $\gamma$ and exp $\left[-i 2 k_{0} \xi\right]$ may be written in terms of their two-dimensional Fourier-Stieltjes amplitudes (a generalization of the Fourier transform for random functions; see Yaglom $[8])$ as

$$
\gamma\left(\rho^{\prime}\right)=\int d \gamma(\mathbf{K}) e^{i \mathrm{~K} \cdot \rho^{\prime}}
$$

and

$$
e^{-i 2 k_{0} \xi\left(\rho_{1}+\rho^{\prime}\right)}=\int d B(\mathbf{K}) e^{i \mathrm{~K} \cdot\left(\rho_{1}+\rho^{\prime}\right)} .
$$

Substituting (9) and (10) into (8), we obtain the relationship 
between the random amplitudes $d \gamma$ and $\mathrm{dB}$ in the form

$$
d \gamma(\mathbf{K})=\frac{-i k_{0} C_{0}}{2 \pi z^{\prime} H_{0}} \int_{A} d^{2} \rho_{1} \frac{e^{i 2 k_{0} r_{0}+i \mathbf{K} \cdot \rho_{1}}}{r_{0}} \mathrm{~dB}(\mathbf{K}) .
$$

Equation (11) demonstrates that $d \gamma$, which can be related to the phase fluctuations by means of (7), is simply the random spectral amplitude $\mathrm{dB}(\mathrm{K})$, which depends upon the waveheight variations times a weighting function $I(\mathrm{~K})$, that is,

$$
d \gamma(\mathbf{K})=I(\mathbf{K}) \mathrm{dB}(\mathbf{K}),
$$

where

$$
I(\mathbf{K})=\frac{-i k_{0} C_{0}}{\left(2 \pi z^{\prime}\right) H_{0}} \int_{A} d^{2} \rho_{1} \frac{e^{i 2 k_{0}} \sqrt{\rho_{1}^{2}+z^{\prime 2}}+i K \cdot \rho_{1}}{\sqrt{\rho_{1}^{2}+z^{\prime 2}}} .
$$

For a circular illumination pattern of diameter $D$, substitute $\mathbf{K}=(K, \theta)$ and integrate over angles

$$
I(K)=\frac{-i k_{0} C_{0}}{z^{\prime} H_{0}} \int_{0}^{D / 2} d \rho_{1} \rho_{1} \frac{e^{i 2 k_{0} \sqrt{\rho_{1}^{2}+z^{\prime 2}}}}{\sqrt{\rho_{1}^{2}+z^{\prime 2}}} J_{0}\left(K \rho_{1}\right),
$$

where $I(K)$ is now a function of only the magnitude of the two-dimensional wavenumber $K$.

From (12) and (14) we can calculate the power spectral density of $d \gamma$ in terms of the power spectral density of $\mathrm{dB}$ and ultimately relate the phase statistics to wave-height statistics. First, we multiply (12) by its complex conjugate

$$
\left\langle d \gamma\left(\mathbf{K}_{1}\right) d \gamma^{*}\left(\mathbf{K}_{2}\right)\right\rangle=I\left(\mathbf{K}_{1}\right) I^{*}\left(\mathbf{K}_{2}\right)\left\langle\mathrm{dB}\left(\mathbf{K}_{\mathbf{1}}\right) \mathrm{dB} *\left(\mathbf{K}_{\mathbf{2}}\right)\right\rangle,
$$

and from the definition (10), calculate the expression in the angle brackets on the right side of (15), that is

$$
\begin{aligned}
& \left\langle e^{-i 2 k_{0}\left[\xi\left(\rho_{1}\right)-\xi\left(\rho_{2}\right)\right]}\right\rangle \\
& \quad=\int e^{i \mathbf{K}_{1} \cdot \rho_{1}} \int e^{-i \mathbf{K}_{2} \cdot \rho_{2}}\left\langle\mathrm{~dB}\left(\mathrm{~K}_{1}\right) \mathrm{dB} *\left(\mathrm{~K}_{2}\right)\right\rangle .
\end{aligned}
$$

If $\xi$ is assumed to be a zero-mean statistically homogeneous Gaussian random variable, which is a reasonable assumption for the wave-height fluctuations [2], it is not difficult to show that [9]

$$
\left\langle e^{-i 2 k_{0}\left[\xi\left(\rho_{1}\right)-\xi\left(\rho_{2}\right)\right]}\right\rangle=e^{-2 k_{0} D^{2}\left(\rho_{1}-\rho_{2}\right)},
$$

where $D_{\xi}$ is the so-called structure function of the wave-height variations defined by

$$
\begin{aligned}
D_{\xi}\left(\rho_{1}-\rho_{2}\right) & =\left\langle\left[\xi\left(\rho_{1}\right)-\xi\left(\rho_{2}\right)\right]^{2}\right\rangle \\
& =2 \sigma_{\xi}^{2}\left[1-R_{\xi}\left(\rho_{1}-\rho_{2}\right)\right],
\end{aligned}
$$

and $\sigma_{\xi}^{2}$ and $R_{\xi}$ are, respectively, the variance and the spatial correlation function of the wave height. Substituting this result into (16), we obtain

$$
\begin{aligned}
& e^{-2 k k_{0}^{2} D_{\xi}\left(\rho_{1}-\rho_{2}\right)} \\
& =\int e^{i \mathrm{~K}_{1} \cdot \rho_{1}} \int e^{-i \mathrm{~K}_{1} \cdot \rho_{2}}\left\langle\mathrm{~dB}\left(\mathrm{~K}_{1}\right) \mathrm{dB}^{*}\left(\mathrm{~K}_{2}\right)\right\rangle .
\end{aligned}
$$

On the left side of (19) we have a function of $\left(\rho_{1}-\rho_{2}\right)$ alone (a consequence of the statistical homogeneity assumption). In order to have a similar dependence on the right side, the quantity in the angle brackets must have the form

$$
\left\langle\mathrm{dB}\left(\mathbf{K}_{1}\right) \mathrm{dB} *\left(\mathbf{K}_{2}\right)\right\}=F\left(\mathbf{K}_{1}\right) \delta\left(\mathbf{K}_{\mathbf{1}}-\mathbf{K}_{\mathbf{2}}\right) d^{2} \mathbf{K}_{\mathbf{1}} d^{2} \mathbf{K}_{\mathbf{2}} .
$$

Substituting this result into (19), we find that $F$ satisfies the relation

$$
e^{-2 k_{0}^{2} D_{\xi}\left(\rho_{1}-\rho_{2}\right)}=\int d^{2} \mathrm{~K}_{2} F\left(\mathrm{~K}_{2}\right) e^{i \mathrm{~K}_{2} \cdot\left(\rho_{1}-\rho_{2}\right)}
$$

and of course, by the Fourier inversion theorem

$$
F(\mathbf{K})=\frac{1}{(2 \pi)^{2}} \int d^{2} \rho e^{-2 k_{0}^{2} D_{\xi}(\rho)} e^{-i \mathrm{~K} \cdot \rho} .
$$

Equation (22) has the same form as the classic integral for the average received power that appears in nearly all treatments of plane-wave scatter from rough Gaussian surfaces since Isakovich's [10] pioneering work in 1952. The principal contribution of the subsequent analysis is (1) to show how this result can be extended to spherical-wave scatter via simple transfer-function concepts, and (2) to employ these concepts to derive the statistics of the received signal phase, rather than its average intensity.

By an analogous procedure we can determine the form of the left side of (15). From (9) and (7),

$$
\begin{aligned}
\left\langle\gamma\left(\rho_{1}\right) \gamma^{*}\left(\rho_{2}\right)\right\rangle= & \left\langle e^{i\left[\phi_{1}\left(\rho_{1}\right)-\phi_{1}\left(\rho_{2}\right)\right]}\right\rangle \\
= & \int e^{i \mathbf{K}_{1} \cdot \rho_{1}} \int e^{-i \mathbf{K}_{2} \cdot \rho_{2}} \\
& \times\left\langle d \gamma\left(\mathbf{K}_{1}\right) d \gamma^{*}\left(\mathbf{K}_{2}\right)\right\rangle
\end{aligned}
$$

Again, if it is assumed that the phase fluctuations $\phi_{1}$ are normally distributed, we can take recourse to the theorem for Gaussian random variables as in the steps leading to (17); then,

$$
\begin{aligned}
\left\langle e^{\left.i\left[\phi_{1}\left(\rho_{1}\right)-\phi_{1}\left(\rho_{2}\right)\right]\right\rangle=}\right. & e^{-1 / 2 D_{\phi}\left(\rho_{1}-\rho_{2}\right)}=\int e^{i \mathbf{K}_{1} \cdot \rho_{1}} \\
& \cdot \int e^{-i \mathbf{K}_{2} \cdot \rho_{2}\left\langle d \gamma\left(\mathbf{K}_{1}\right) d \gamma^{*}\left(\mathbf{K}_{2}\right)\right\rangle,}
\end{aligned}
$$

where $D_{\phi}$ is the structure function of the phase fluctuations. In order to have the $\rho_{1}-\rho_{2}$ dependence on the right side of (24),

$$
\left\langle d \gamma\left(\mathbf{K}_{1}\right) d \gamma^{*}\left(\mathbf{K}_{2}\right)\right\rangle=G\left(\mathbf{K}_{1}\right) \delta\left(\mathbf{K}_{\mathbf{1}}-\mathbf{K}_{2}\right) d^{2} \mathbf{K}_{1} d^{2} \mathbf{K}_{\mathbf{2}},
$$


$G$ must satisfy

$$
e^{-1 / 2 D_{\phi}(\rho)}=\int d^{2} \mathbf{K} G(\mathrm{~K}) e^{i \mathrm{~K} \cdot \rho}
$$

and the inverse relation

$$
G\left(\mathbf{K}_{\mathbf{1}}\right)=\frac{1}{(2 \pi)^{2}} \int d^{2} \rho e^{-1 / 2 D_{\phi}(\rho)} e^{-i \mathbf{K}_{1} \cdot \rho}
$$

We now can substitute (25) and (20) into (15) and obtain

$$
G\left(\mathbf{K}_{\mathbf{1}}\right)=\left|I\left(K_{1}\right)\right|^{2} F\left(\mathrm{~K}_{\mathbf{1}}\right)
$$

The result of the steps leading to (28) is to transform the scattering problem to the spatial frequency domain. The conventional approach to sea scatter calculations is to take a form of the physical optics integral (5) and to determine the desired statistics of $H$ by approximating the integral, e.g., assuming plane wave incidence, applying stationary phase, etc., using information about typical surface roughness parameters, and averaging. Equation (28) demonstrates that the statistics of $H$ may be determined exactly by transforming the scattering problem into the spatial frequency domain. Here the linear system aspect of the problem is emphasized, and the spectral statistics of the received field $G(K)$ factor conveniently into two terms, separating the spectral quantity that depends on the wave-height statistics for plane-wave incidence $F(\mathbf{K})$ from a quantity $\mid I \mathrm{i}^{2}$ that depends solely on the propagation geometry. Equation (28) is the equation of a linear system in the spatial frequency domain that describes how the input spectrum (the angular spectrum of scattered plane waves leaving the sea surface $F$ ), is multiplied by a transfer function $|I|^{2}$ so that the resultant $G=|I|^{2} F$ is the angular spectrum of scattered waves at the receiver. Examination of (13), which is the definition of $I$, reveals that it is also the diffraction integral for the field backscattered from a sinusoidal surface irregularity of wavenumber $K$ whose amplitude is small in terms of the radio wavelength, observed at a distance $z^{\prime}$ from the surface. Therefore, the physical interpretation of $|I|^{2}$ is that it describes the angular distribution of power scattered by a single Fourier surface component in the "slightly rough" or Rayleigh limit [2], [11], [12]. This result could have been anticipated, since from linear system theory, $|I|^{2}$ is the transfer function or the frequency response of the medium.

Equation (28) can be solved formally to give the waveheight statistics by using the Fourier transform. By inserting the definition of $F$ from (22) into (28), we obtain

$$
e^{-2 k_{0}{ }^{2} D_{\xi}(\rho)}=(2 \pi)^{2} \int d^{2} K_{1} e^{i K_{1} \cdot \rho}\left|I\left(K_{1}\right)\right|^{-2} G\left(K_{1}\right)
$$

or, by substituting the definition of $G$,

$$
\begin{array}{r}
D_{\xi}(\rho)=-\frac{1}{2 k_{0}^{2}} \ln \left\{\int d^{2} \mathbf{K}_{1} e^{i \mathbf{K}_{1} \cdot \rho}|I|^{-2}\right. \\
\left.\cdot \int d^{2} \rho_{1} e^{-i \mathbf{K}_{1} \cdot \rho_{1}-1 / 2 D_{\phi}\left(\rho_{1}\right)}\right\} .
\end{array}
$$

Without any further approximation we can find the waveheight structure function, a quantity directly related to the power spectral density, by measuring the structure function of the phase fluctuations of the backscattered signal, computing the Fourier transform of exp $\left[-\frac{1}{2} D_{\phi}\right]$, multiplying by $|I|^{-2}$ [found from (14)], Fourier transforming the result and, finally, computing the logarithm. In a practical case, we would use the satellite or airplane velocity $\mathrm{v}$ times the time lag $\tau$ as the vector $\rho_{1}$. In this instance, the $D_{\phi}$ would be computed from the recorded time series of the phase fluctuations, where

$$
D_{\phi}(\tau)=\left\langle\left[\phi_{1}(t)-\phi_{1}(t+\tau)\right]^{2}\right\rangle
$$

and the transforms in (30) would reduce to their one-dimensional analogs.

\section{APPROXIMATIONS}

In this section, we will show that, for certain propagation parameters, $(28)$ and $(30)$ reduce to an especially simple form. The quantity $I(K)$ is defined by

$$
I(K)=\frac{-i k_{0} C_{0}}{z^{\prime} H_{0}} \int_{0}^{D / 2} d \rho_{1} \rho_{1} \frac{e^{i 2 k} 0 \sqrt{\rho_{1}^{2}+z^{\prime 2}}}{\sqrt{\rho_{1}^{2}+z^{\prime 2}}} J_{0}\left(K \rho_{1}\right),
$$

where, from geometrical optics, $H_{0}=C_{0} \exp \left[i 2 k_{0} z^{\prime}\right] /\left(2 z^{\prime}\right)$ is the field observed at the receiver in the absence of surface roughness. ${ }^{1}$ If we change variables to $y=\sqrt{\rho^{2}+z^{\prime 2}}$ and note from Fig. 1 that $D / 2=z^{\prime} \tan \theta_{0},(32)$ reduces to

$$
I(K)=-i 2 k_{0} e^{-i 2 k} 0^{z^{\prime}} \int_{z^{\prime}}^{z^{\prime} \tan \theta 0} d y e^{i 2 k_{0} y} J_{0}\left(K \sqrt{y^{2}-z^{\prime 2}}\right) .
$$

After considerable manipulation, this integral can be evaluated in terms of Lommel's functions of two variables

$$
\begin{aligned}
I(K)= & e^{i 2 k z^{\prime}\left(\sec \theta_{0}-1\right)}\left[e^{i 2 k_{0} z^{\prime}\left(1-\sec \theta_{0}\right)-i\left(K^{2} z^{\prime} / 4 k_{0}\right)}\right. \\
& -J_{0}\left(K z^{\prime} \tan \theta_{0}\right)+i U_{1}\left(\frac{K^{2} z^{\prime}}{2 k_{0}}, K z^{\prime} \tan \theta_{0}\right) \\
& \left.+U_{2}\left(\frac{K^{2} z^{\prime}}{2 k_{0}}, K z^{\prime} \tan \theta_{0}\right)\right]
\end{aligned}
$$

where the Lommel function is defined by

$$
U_{\nu}(\omega, z)=\sum_{m=0}^{\infty}(-1)^{m}\left(\frac{\omega}{z}\right)^{\nu+2 m} J_{\nu+2 m}(z)
$$

A plot of $|I|^{2}$ from (34) is shown in Fig. (2). There are three important features of this curve. First, from $(34),|I(0)|^{2}=$

1 The spherical nature of the reflecting earth can be accounted for, if necessary, by replacing $z^{\prime}$ in the denominator by $z^{\prime}+a$, where $a$ is the radius of the earth. 


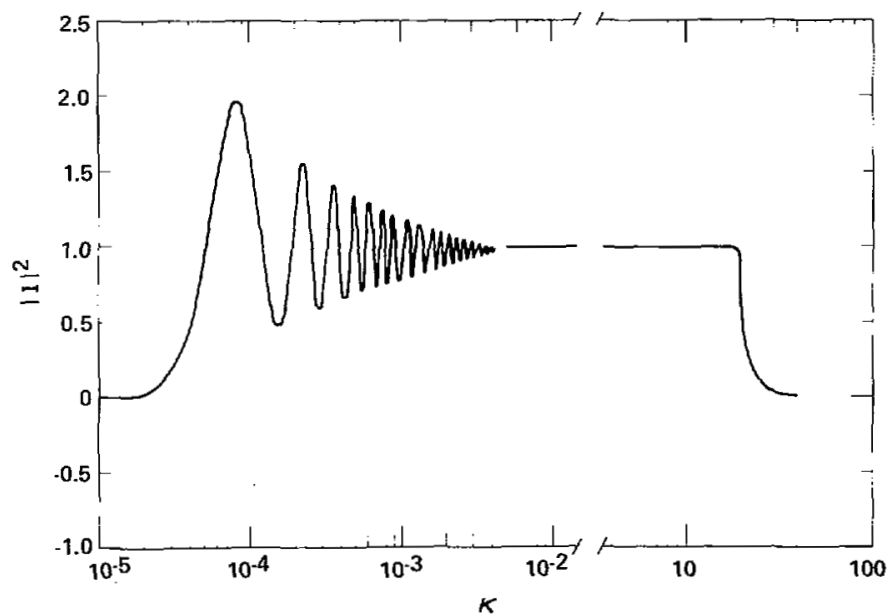

Fig. 2. Plot of transfer function $|I|^{2}$ versus $K$. For $K / 2 k_{0}=\sin \theta$, $|I|^{2}$ is also angular spectrum of plane waves scattered from truncated Fourier component of surface roughness of wavenumber $K$.

$4 \sin ^{2}\left[k_{0} z^{\prime}\left(1-\sec \theta_{0}\right)\right]$, the scattering cross section of a circular plate of diameter $2 z^{\prime} \tan \theta_{0}$. Second, the curve asymptotically approaches its final value of unity beginning at $K \approx 2\left(z^{\prime} \tan \theta_{0}\right)^{-1}$, indicating the irregularities in the surface roughness that are larger than, or of the same order as, the beam diameter are severely affected by the truncated illumination of the beam pattern; whereas, higher spatial frequencies are not. Third, the cutoff of $|I|^{2}$ occurs at a spatial wavenumber $K=2 k_{0}$ tan $\theta_{0}$. These three characteristics have simple physical interpretations. The quantity $|I|^{2}$ describes the first-order Bragg scattering properties of different Fourier components of the surface roughness that are truncated by the finite illumination pattern of the transmitter. For very low spatial frequencies $(K \rightarrow 0)$ the irregular surface resembles a flat, circular plate so that the power at $K=0$ should be proportional to the scattering cross section of that particular geometry. As observed, the scattering properties of shortwavelength Fourier components of surface roughness (much less than the beam diameter $2 z^{\prime} \tan \theta_{0}$ ) are essentially unaffected by the truncated illumination pattern, whereas larger spatial wavelengths will have their scattering lobes considerably broadened. Finally, from the Bragg theory, surface irregularities of spatial wavenumber $K$ will backscatter energy at an angle $\theta=\sin ^{-1}\left[K / 2 k_{0}\right]$. Clearly, when $K / 2 k_{0}>\tan \theta_{0}$ most of the energy will be scattered out of the receiving cone. This accounts for the precipitous decrease of $\left.I I\right|^{2}$ beyond the point $K=2 k_{0} \tan \theta_{0}$.

A useful engineering approximation for $|I|^{2}$ can be obtained from (34):

$$
\left.I I(K)\right|^{2}=\left\{\begin{array}{c}
1+J_{0}^{2}\left(K z^{\prime} \tan \theta_{0}\right) \\
-2 \cos \left[2 k_{0} z^{\prime}\left(1-\sec \theta_{0}\right)\right] J_{0}\left(K z^{\prime} \tan \theta_{0}\right) \\
\text { for } K \leqslant 2 k_{0} \tan \theta_{0} \\
0, \quad \text { for } K>2 k_{0} \tan \theta_{0}
\end{array}\right.
$$

From Fig. (2), we see that $|I|^{2} \approx 1$ in the region $\left(z^{\prime} \tan \theta_{0}\right)^{-1} \ll K \leqslant 2 k_{0} \tan \theta_{0}$. For typical satellite parameters $\theta_{0} \sim 0.05, k_{0} \cong 200 \mathrm{~m}^{-1}$, and $z^{\prime} \sim 10^{6} \mathrm{~m}$, we have $2 \times 10^{-5} \mathrm{~m}^{-1} \ll K \lesssim 20 \mathrm{~m}^{-1}$. Over this broad spectral range the angular spectrum of plane waves arriving at the receiver $G(K)$ is identical to the angular spectrum of scattered waves just above the ocean surface $F(K)$.

Because there is negligible area under $|I|^{2}$ in the region $0 \leqslant K \leqslant 10\left(z^{\prime} \tan \theta_{0}\right)^{-1}$ compared with the region $10\left(z^{\prime} \tan \theta_{0}\right)^{-1} \leqslant K \leqslant 2 k_{0}$ tan $\theta_{0}$ for a typical satellite geometry, we make a negligible error by assuming that $|I|^{2}$ is roughly constant throughout the entire region $0 \leqslant K \leqslant$ $2 k_{0} \tan \theta_{0}$. From (28) we see that $G(\mathbf{K})=F(\mathbf{K})|I|^{2}$. If $F(K)$ has no significant power beyond $K=2 k_{0} \tan \theta_{0}$, then $G(\mathbf{K})=$ $F(\mathrm{~K})$, and from their respective definitions, [(22) and (27)] $D_{\phi}(\rho)=4 k_{0}{ }^{2} D_{\xi}(\rho)$. Hence, the wave-height statistics are proportional to the phase statistics.

To estimate the bandwidth of $F(K)$, note that $F(K)$ is the power spectral density of $\exp \left[i 2 k_{0} \xi\right]$ with $\xi$ a random variable. A reasonable estimate of the bandwidth of a phase modulated signal is the maximum instantaneous spatial frequency. For a phase modulated wave exp $[i \phi(x)]$ the bandwidth $\delta \leqslant(\partial \phi / \partial x)_{\max }$. In the case of phase modulation due to ocean waves $\delta \sim 2 k_{0} s$ where $s$ is the rms wave slope. Again, to minimize spectral distortion, the bandwidth of $F(K)$ must be less than the spectral width of $|I|^{2}$, i.e.,

$$
\tan \theta_{0}>s
$$

For $s \cong 0.04$ a half-beamwidth of approximately three degrees will satisfy (36). Under this approximation, the phase statistics and wave height statistics are proportional, that is

$$
D_{\phi}(\rho)=4 k_{0}^{2} D_{\xi}(\rho) \text {. }
$$

\section{CONCLUSIONS}

We have shown that, for reasonable microwave propagation parameters $\left(f_{0} \sim 10 \mathrm{GHz}, k_{0} \sim 200 \mathrm{~m}^{-1}, \theta_{0} \sim 0.05 \mathrm{rad}\right.$ antenna half-beamwidth), the phase structure function ${ }^{2}$ can be written directly in terms of the wave-height structure function giving

$$
D_{\phi}(\rho)=4 k_{0}^{2} D_{\xi}(\rho)
$$

This also implies the following relationship between the signal phase spatial spectrum $S_{\phi}(\mathbf{K})$ and the two-dimensional waveheight spatial spectrum $S(\mathbf{K})$ :

$$
S_{\phi}(\mathrm{K})=4 k_{0}{ }^{2} S(\mathrm{~K})
$$

A satellite traversing a line can measure the above quantities only along a single direction, i.e., $\mathbf{K}=K \cos \alpha \hat{x}+K \sin \alpha \hat{y}$, where $\alpha$ is the nadir-pointing track angle with respect to an arbitrary $x-y$ coordinate geometry at the earth's surface. This unidirectional wave spectral measurement is identical to that obtained from an airborne laser profilometer, where the received signal phase (or time of flight) varies in direct proportion to the height of the sea as the instrument "profiles" the ocean waves. The important conclusion to be drawn here is that the final spectral results (40) are identical for the laser profilometer and the microwave nadir-pointing phase-measuring radar, even though the scattering mechanisms are entirely different. In the former case, the reflection occurs from an

2 Assumed in this discussion is the continuous measurement of phase beyond a single $0-360^{\circ}$ interval. 
infinitesimally small moving spot on the surface, whereas in the latter the scatter originates from a continuous, rough sea patch tens of kilometers in diameter.

A final, obvious result, is implied by (39) and (40), relating the rms sea wave height $k$ to the rms signal phase $\sigma_{\phi}$ :

$$
\sigma_{\phi}=2 k_{0} h
$$

\section{APPENDIX I}

In the derivation of (7), we ignore the contributions of the amplitude fluctuations. If these fluctuations are retained (7) would have read

$$
\gamma\left(\rho^{\prime}\right)=e^{x\left(\rho^{\prime}\right)+i \phi_{1}\left(\rho^{\prime}\right)},
$$

where $x=\ln \left(A / A_{0}\right)$ is the logarithm of the relative amplitude of the received signal. The contribution of $x$ in the final result (38) can be estimated by computing $\left\langle\gamma \gamma^{*}\right\rangle$. If we assume that fluctuations in $x$ and $\phi_{1}$ are uncorrelated, then

$$
\left\langle\gamma\left(\rho^{\prime}\right) \gamma^{*}\left(\rho^{\prime}+\rho\right)\right\rangle=\left\langle e^{x\left(\rho^{\prime}\right)+x\left(\rho^{\prime}+\rho\right)}\right\rangle\left\langle e^{i\left[\phi_{1}\left(\rho^{\prime}\right)-\phi_{1}\left(\rho^{\prime}+\rho\right)\right]}\right\rangle .
$$

From (24) the second factor of (A2) can be written in terms of the phase structure function, and we obtain

$$
\left\langle\gamma\left(\rho^{\prime}\right) \gamma^{*}\left(\rho^{\prime}+\rho\right)\right\rangle=\left\langle e^{x\left(\rho^{\prime}\right)+x\left(\rho^{\prime}+\rho\right)}\right\rangle e^{-1 / 2 D_{\phi}(\rho)}
$$

To estimate the error in ignoring the first factor we compute the indicated expected value in two cases, when the $x$ 's are completely correlated and then, when they are completely uncorrelated. If the amplitude fluctuations are Rayleigh distributed, that is,

$$
P(A)=\frac{A}{\sigma^{2}} e^{-A^{2} /\left(2 \sigma^{2}\right)},
$$

then it is easy to prove that

$$
P(x)=2 e^{2 x} e^{-e^{2 x}} .
$$

From (A5), we calculate the expected value for the case of perfect correlation,

$$
\left\langle e^{2 x}\right\rangle=1,
$$

and for the case of uncorrelated random variables,

$$
\left\langle e^{x} X e^{x}\right\rangle=\pi / 4
$$

Therefore, throughout the whole range of displacements $\rho$,

$$
\pi / 4 \leqslant\left\langle e^{x\left(\rho^{\prime}\right)+x\left(\rho^{\prime}+\rho\right)}\right\rangle \leqslant 1
$$

Equation (A4) is the error attributed to ignoring amplitude fluctuations. To compute the ultimate error in the estimate of the phase fluctuations, we note that (A4) enters into the final calculation (38) in the form

$$
D_{\phi}(\rho)=4 k_{0}^{2} D_{\xi}(\rho)+2 \ln \left[\left\langlee^{\left.\left.x\left(\rho^{\prime}\right)+x\left(\rho^{\prime}+\rho\right)\right\rangle\right]} .\right.\right.
$$

The second term ranges between zero and $2 \ln (\pi / 4)=-0.48$ as $\rho$ increases. In the same range of $\rho$, the first term goes from zero to $4 k_{0}^{2} \sigma_{\xi}^{2}$, where $\sigma_{\xi}^{2}$ is the waveheight variance. For a rough sea surface $\sigma_{\xi} \sim 1$ and $4 k_{0}^{2} \sigma_{\xi}^{2} \sim 1.6 \times 10^{5}$. Clearly, the error introduced by ignoring amplitude fluctuations is insignificant.

\section{APPENDIX II}

As pointed out by the reviewers, there is an additional more fundamental interpretation of (28) in terms of the coherence function of the wave field upon reception. Actually, the coherence history is recorded in (28). Initially, the wave emitted by the antenna of aperture size $d_{0}$ illuminates the sea surface with a wave that is coherent over its illuminated spot diameter $D \sim 2 z^{\prime} /\left(k_{0} d_{0}\right)$. Immediately upon reflection the wave phase front has the same corrugations as the sea surface itself; therefore, the coherence properties just above the sea surface are determined by the properties of the rough surface. The coherence function of the wave back at the receiver will be a convolution of the surface statistics and purely geometrical propagation factors such as $\lambda, z^{\prime}$, and $d_{0}$.

To illustrate these points, we return to the Fourier transformed version of (28)

$$
\begin{gathered}
M(\rho) \equiv \frac{\left\langle H\left(\rho^{\prime}\right) H^{*}\left(\rho^{\prime}+\rho\right)\right\rangle}{\left|H_{0}\right|^{2}}=\int d^{2} \mathbf{K}_{1} e^{i K_{1} \cdot \rho}\left|I\left(K_{1}\right)\right|^{2} \\
\cdot \int d^{2} \rho_{1} \frac{e^{-2 k_{0} D^{2} D_{\xi}\left(\rho_{1}\right)-i K_{1} \cdot \rho_{1}}}{(2 \pi)^{2}}
\end{gathered}
$$

where $M$ is the mutual coherence function (MCF) of the received field. (Note $e^{-2 k 0^{2} D_{\xi(\rho 1)}}$ is the MCF of the scattered field at the sea surface immediately after scattering). For illustrative purposes only, we assume that $|I|^{2}$ is a Gaussian function of the form

$$
|I|^{2} \sim e^{-K^{2} /\left(2 k_{0} \tan \theta_{0}\right)^{2}},
$$

in line with the behavior of $|I|^{2}$ exhibited in its approximate version (36). We now approximate $D_{\xi}\left(\rho_{1}\right)=2 \sigma_{\xi}^{2}\left[1-R_{\xi}\left(\rho_{1}\right)\right]$ by a quadratic function of $\rho_{1}$, that is, $D_{\xi}\left(\rho_{1}\right) \approx 2 \sigma_{\xi}^{2} \rho_{1}^{2} / \rho_{s}^{2}$ where from the definition of $R_{\xi}\left(\rho_{1}\right), \rho_{s}$ is the correlation length of the irregular sea surface that has a wave-height variance $\sigma_{\xi}^{2}$. It is not difficult to show that this assumption reduces the coherence function of the wave just as it leaves the sea surface to a form consistent with Barrick's [6], [13] stationary phase solution in terms of rms wave slope, that is,

$$
\begin{aligned}
e^{-2 k_{0} D^{2} D_{\xi}\left(\rho_{1}\right)} & =e^{-4 k_{0}{ }^{2} \sigma_{\xi}^{2}\left[1-R \xi^{2}\left(\rho_{1}\right)\right]} \\
& =e^{-4 k_{0}{ }^{2} \sigma_{\xi}^{2}\left(\rho_{1}{ }^{2} / \rho_{s} 2\right)} .
\end{aligned}
$$

To proceed further, we note that $\rho_{s}=2 \sigma_{\xi} s^{-1}$, where $s$ is the rms surface slope, and therefore

$$
e^{-2 k_{0}{ }^{2} D_{\xi}\left(\rho_{1}\right)} \equiv e^{-\left(\rho_{1} / \rho_{0}\right)^{2}}=e^{-k_{0}{ }^{2} s^{2} \rho_{1} 2}
$$


where from our development $\rho_{0 s}$ is interpreted as the coherence length of the wave just as it leaves the sea surface. The form of (A13) is now identical to that of Barrick's solution. If we insert (A13) and (A11) into (A10) we obtain the result

$$
M(\rho) \propto \exp \frac{-\rho^{2}}{4}\left[\frac{1}{k_{0}^{2} \tan ^{2} \theta_{0}}+\frac{1}{k_{0}^{2} s^{2}}\right]^{-1},
$$

so the coherence length of the received field, defined roughly by $M\left(\rho_{0}\right)=e^{-1}$, becomes

$$
\rho_{0}=2\left[\frac{1}{k_{0}^{2} \tan ^{2} \theta_{0}}+\frac{1}{\left(k_{0} s\right)^{2}}\right]^{1 / 2} .
$$

To put the result in final form, note that $\tan \theta_{0} \sim 2 /\left(k_{0} d_{0}\right)$, so that

$$
\rho_{0}=\left[d_{0}^{2}+\frac{4}{\left(k_{0} s\right)^{2}}\right]^{1 / 2} .
$$

Note that as the coherence length of the field at the surface $\rho_{0 s}=\left(k_{0} s\right)^{-1} \rightarrow 0$, that is, the scattering is completely incoherent, we obtain the result attributable to the Van CittertZernike theorem; namely, you obtain independent samples of the scattered field each time the receiver aperture moves its diameter. The result (A16), the modified Van Cittert-Zernicke theorem, shows that in partially coherent scattering, which is the case applicable to the sea scatter problem, there is an additional surface-dependent term that contributes to the coherence length of the received field. In the general case $d_{0} \sim 4\left(k_{0} s\right)^{-1}$ so that a more complicated inversion procedure than that suggested by (38) will be necessary (see comments about (30)).

Finally, as a caution, (38) demonstrates that the phase correlation length is the same as the wave-height correlation length. This should not be confused with the coherence length of the field which is given by (A16) and is considerably smaller. The field decorrelates in a distance much shorter than the distance over which the phase decorrelates.

\section{REFERENCES}

[1] D. E. Barrick, "First-order theory and analysis of MF/HF/VHF scatter from the sea," IEEE Trans. Antennas Propagat., vol. AP-20, pp. 2-10, Jan. 1972.

[2] D. E. Barrick, "Remote sensing of sea state by radar," in Remote Sensing of the Troposphere, V. E. Derr, Ed. Washington, DC: U.S. Government Printing Office, 1972, pp. 12.1-12.24.

[3] P. Wadhams, "Airborne laser profiling of swell in an open ice field," J. Geophysical Res., vol. 80, pp. 4520-4528, 1975.

[4] J. T. McGoogan, "Satellite altimetry applications," IEEE Trans. Microwave Theory Tech., vol. MTT-23, pp. 970-978, 1975.

[5] C. T. Swift, and W. L. Jones, Jr., "Satellite radar scatterometry," 1974 IEEE INTERCON, Technical Papers, Paper No. 34/4, 1974.

[6] D. E. Barrick, "Relationship between slope probability density and the physical optics integral in rough surface scattering," in Proc. IEEE, vol 56, pp. 1728-1729, 1968.

[7] G. T. Ruck, D. E. Barrick, W. D. Stuart, and C. K. Krichbaum, Radar Cross Section Handbook, Vols. I and II. New York: Plenum, 1970, pp. 50-63 and 719-727.

[8] A. M. Yaglom, Introduction to the Theory of Stationary Random Functions. Englewood Cliffs, NJ: Prentice-Hall, 1962, 223.

[9] V. 1. Tatarskii, The Effects of the Turbulent Atmosphere on Wave Propagation. Springfield, VA: National Technical Information Service, $1971,472$.

[10] M. Isakovich, "The scattering of waves from a statistically Iough surface," Zhurnal Eksperimentalnoi Teoreticheskoi Fiziki (USSR), vol. 23, pp. 305-314, 1952. English translation by $M$. Friedman.

[11] Lord Rayleigh, Theory of Sound, Vol. II. London: Macmillan, 1929, pp. 89-96.

[12] S. O. Rice, "Reflection of electromagnetic waves from slightly rough surfaces," in Theory of Electromagnetic Waves, M. Kline, Ed. New York: Interscience, 1951, pp. 351-378.

[13] D. E. Barrick, "Rough surfaces," Chapter 9 of Radar Cross Section Handbook, Vol. II, G. Ruck, Ed. New York: Plenum, 1970, pp. 671-772. 